\title{
Imaging findings in an infant with congenital pul- monary venolobar syndrome (scimitar syndrome)
}

T Westgarth-Taylor, MB ChB, FC Rad Diag (SA)

Department of Radiology, Groote Schuur Hospital, Cape Town

\section{T Kilborn, MB ChB, FRCR}

Department of Radiology, Red Cross War Memorial Children's Hospital, Cape Town

Corresponding author: T Westgarth-Taylor (twestgarthtaylor@gmail.com)

\begin{abstract}
Congenital venolobar syndrome (CVLS) is a rare complex malformation that includes hypoplasia of the lung, partial anomalous pulmonary venous drainage and, in a quarter of patients, associated cardiac anomalies. We present the chest radiograph and multidetector CT findings in a case of congenital venolobar syndrome in an infant, and describe the history of the condition.
\end{abstract}

\section{Introduction}

Congenital venolobar syndrome (CVLS) is a unique and rare form of lobar hypoplasia associated with other anomalies of the pulmonary vessels. ${ }^{1-4}$ The reported incidence is $0.4-0.7 \%$ of all congenital lung anomalies. ${ }^{3}$ The syndrome's variable components include: lung hypoplasia (right more commonly affected than left), partial anomalous pulmonary venous drainage (to right atrium, coronary sinus, inferior vena cava (IVC), or hepatic circulation), absent or small pulmonary artery, systemic arterial supply to the lung, cardiac anomalies (atrial septal defect (ASD), ventricular septal defect (VSD), patent ductus arteriosus (PDA), tetralogy of Fallot, coarctation, hypoplastic left heart or endocardial cushion defects), diaphragmatic hernia, and anomalies of the bony thorax or thoracic soft tissues. ${ }^{2,4}$ Embryologically ,it is thought to represent a primary developmental anomaly of the right lung with secondary anomalous venous drainage. ${ }^{4}$

George Cooper and Raoul Chassinat are credited with the earliest description of the anomaly in the medical literature. In 1836, they individually described single cases of a rare congenital malformation that included venous drainage from the lung beneath the diaphragm. ${ }^{5,6}$ The first authors to use the term 'scimitar' were Halasz et al. in 1956. They referred to a vertical curvilinear vein resembling a scimitar, draining all or a portion of the right lung to the right atrium or more commonly to the IVC.

The first detailed radiographic description of the 'scimitar sign' (the presence of a scimitar vein on a chest radiograph) was by Dotter et al. in 1949; they were also the first to use angiography and cardiac catheterisation to document the anomaly. ${ }^{8}$ The term 'scimitar syndrome' first appears in the 1960 article by Neill et al., referring to the combination of anomalous venous drainage of all or most of the right lung to the right atrium or IVC together with hypoplasia of the right lung and associated with systemic arterial collaterals. ${ }^{9}$ The name of the syndrome has subsequently been refined, and the preferred term 'congenital pulmonary venolobar syndrome' was coined by Dr Ben Felson in 2003. ${ }^{3}$
The clinical presentation of CVLS is variable. Patients may be completely asymptomatic or symptomatic early in life; it depends on the associated cardiac abnormalities and degree of shunting., ${ }^{3,4}$ Surgery is considered in patients who are symptomatic, and requires detailed and accurate preoperative imaging.

\section{Case report}

Our patient presented at 7 weeks of age with a 2-day history of cough and fever, and was initially diagnosed with right-sided pneumonia. At the time of presentation, a murmur was documented.

Chest radiography showed volume loss of the right lung with mediastinal shift to the right and opacification of the right hemithorax. The right lung was therefore difficult to assess and the heart size could not be accurately measured. The left lung showed air trapping and pulmonary plethora (Fig. 1).

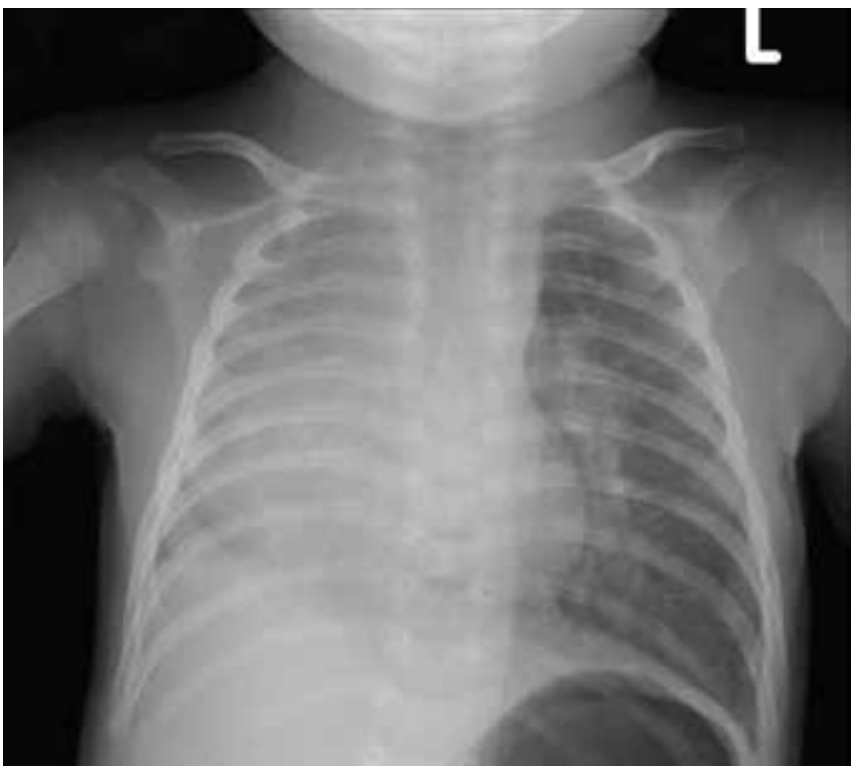

Fig. 1. Frontal chest radiograph showing opacified right hemithorax with mediastinal shift to the right and small right lung when compared with the left. The left lung is hyperinflated and plethoric.

In view of the clinical and radiographic findings, an echocardiogram was performed which revealed multiple cardiac anomalies including a large ASD, small VSD, and dilated right atrium, ventricle and main pulmonary artery. The right and left pulmonary arteries were not well defined. This explained some of the clinical findings; however, the 
volume loss of the right lung remained a concern, and a chest CT was performed to further define the anatomy.

The chest CT was done in the arterial phase and showed a hypoplastic right upper lobe bronchus and consequently also a hypoplastic right lung - this explained the mediastinal shift to the right (Fig. 2). The right pulmonary artery was small, with normal main and left pulmonary arteries (Fig. 3). Assessment of the venous drainage revealed an anomalous right pulmonary vein entering the IVC at the junction with the right atrium - a so-called 'scimitar vein' (Fig. 4). The left pulmonary veins showed normal drainage into the left atrium.

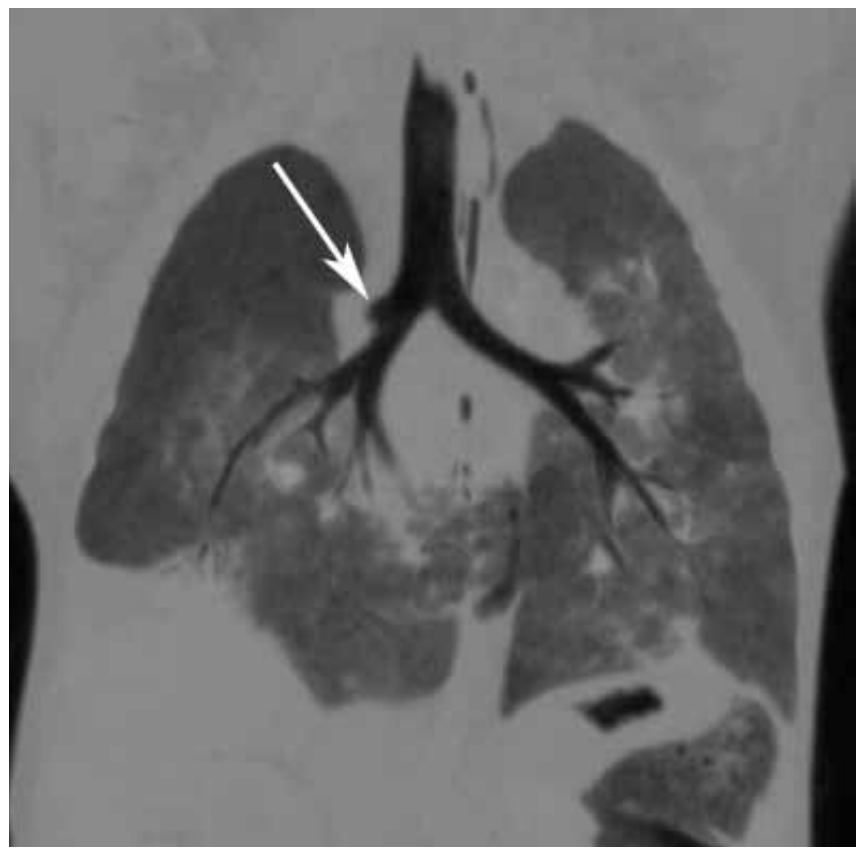

Fig. 2. Coronal minimum intensity projection (MINIP) showing the hypoplastic right upper lobe bronchus and right lung.

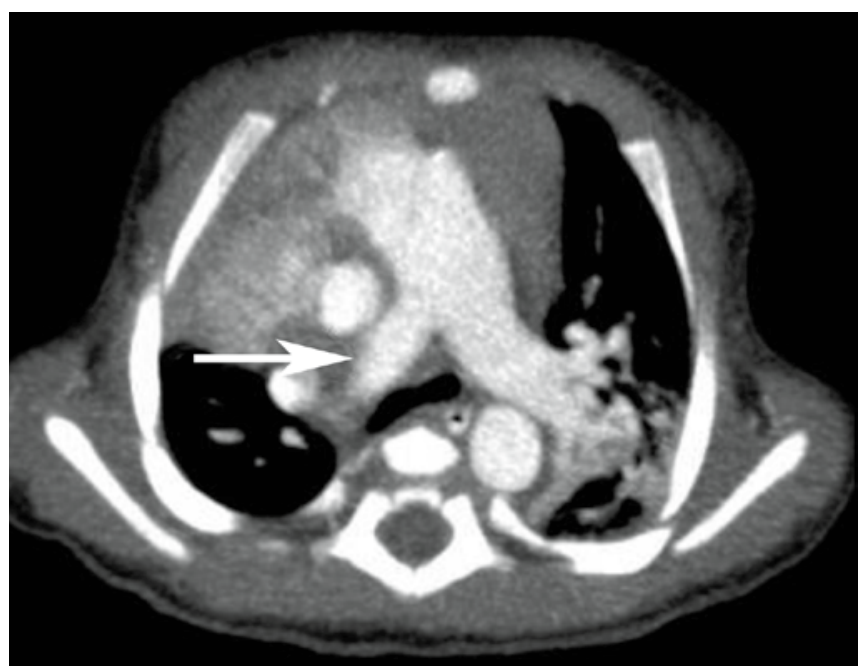

Fig. 3. Axial maximum intensity projection (MIP) showing the small right pulmonary artery (arrow).

The heart was enlarged and the pulmonary vasculature prominent, in keeping with the echo findings of a left to right shunt (ASD and VSD). The study also documented coarctation of the aorta at the level of a PDA, neither of which had been shown on echocardiography (Fig. 5).

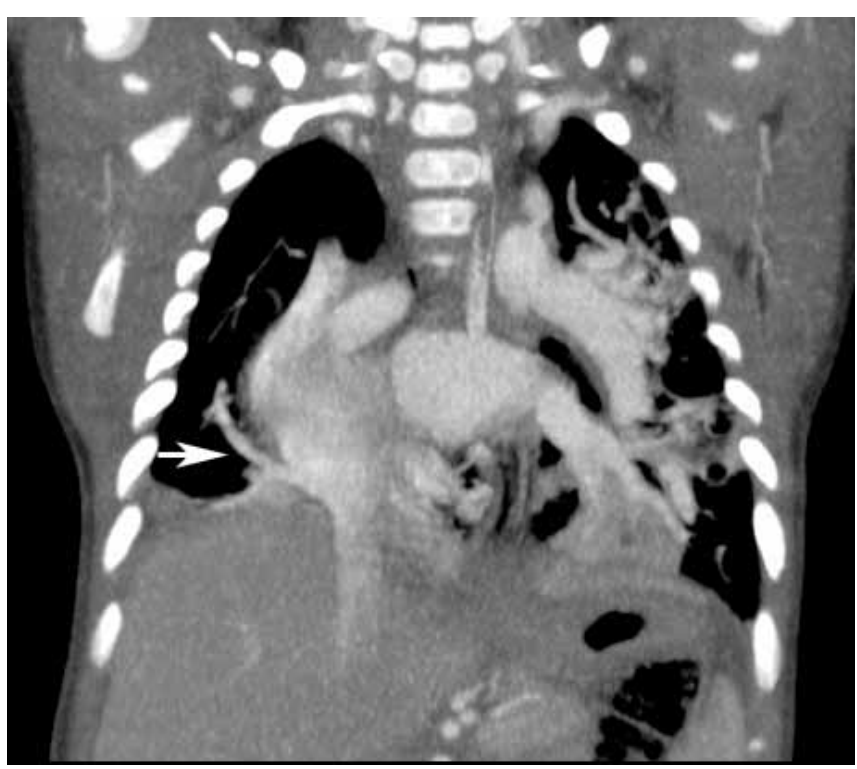

Fig. 4. Coronal MIP showing the scimitar vein (arrow) and its point of insertion into the IVC. Note the plethoric left lung.

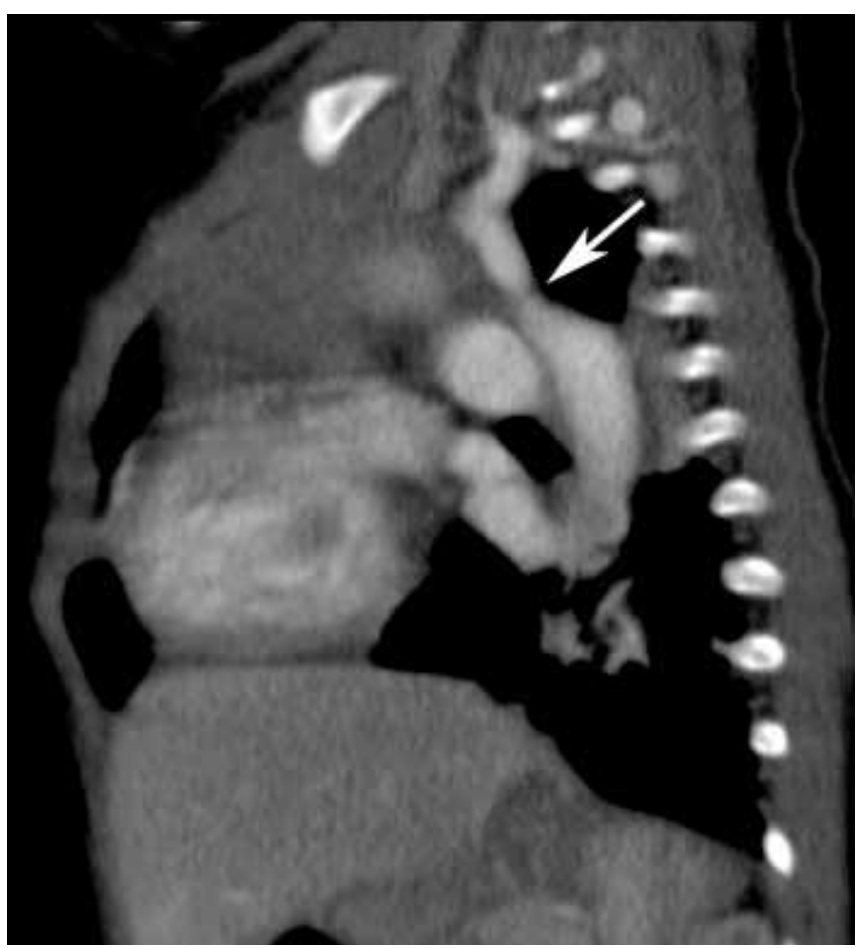

Fig. 5. Oblique sagittal MIP image showing the aortic coarctation (arrow).

\section{Discussion}

Congenital venolobar syndrome refers to a wide spectrum of pulmonary developmental anomalies, which may occur singly or in combination.

Our case highlights the main components of CVLS i.e. hypoplastic right lung with a small right pulmonary artery and anomalous pulmonary venous drainage of the right lung. In $25 \%$ of cases there is an associated cardiac abnormality, most commonly septal defects, as in our case. ${ }^{3,4}$ Coarctation of the aorta is associated with CVLS, but is uncommon and the actual incidence unknown. Our patient did not exhibit co-existent diaphragmatic or soft-tissue abnormalities. 
The present case also demonstrates the diagnostic difficulties that may be encountered with CVLS. It is a rare disorder, not often diagnosed at initial presentation. Mediastinal shift and dextroposition of the heart on plain film are indicators of pulmonary hypoplasia, which warrants further investigation. This is particularly important if there is a co-existing cardiac anomaly or a suspicion of the scimitar sign on the radiograph. In these patients, the venous drainage and arterial supply of the lung need to be defined in order to direct management. ${ }^{4}$

Echocardiography is the initial additional imaging method used in suspected cardiac anomalies. However, in the case of infants, small or absent pulmonary arteries and anomalous venous drainage can be very difficult to define. ${ }^{10}$ Angiography has traditionally been used as an adjunct to echocardiography in the investigation of suspected pulmonary arterial and venous anomalies but is time-consuming, invasive, uses radiation, requires general anaesthetic and can have complications related to arterial access.

More recently, MDCT has been shown to be useful in accurately identifying both the pulmonary arteries and anomalous pulmonary venous return in CVLS., ${ }^{4,10,11}$ As well as the source images, 3D reconstructed images are particularly helpful in depicting the entire course of the anomalous scimitar vein and its point of drainage as well as any systemic arterial supply to the lung. ${ }^{4}$ MDCT also eloquently defines the tracheo-bronchial tree and associated lung abnormalities, as well as the cardiac and other non-vascular anomalies. ${ }^{1,4,10}$ MDCT has the advantages of not requiring an anaesthetic or arterial access and is a fast, non-invasive investigation that has assumed a pivotal role in evaluating patients with congenital lung anomalies. The radiation dose delivered at MDCT should be modified by using dose reduction techniques and paediatric protocols.

Magnetic resonance angiography using contrast-enhanced 3D MRI has also been shown to have a good correlation with surgical findings and does not share the radiation burden of CT and angiography. ${ }^{12}$ However, it does require expertise that is not readily available at most institutions - ours included.
CVLS in isolation is compatible with life; symptoms in these patients are usually due to the associated cardiac abnormalities, and the severity thereof determines prognosis. ${ }^{5,6}$ When patients are symptomatic, surgical correction of the cardiac abnormality along with scimitar vein ligation or embolisation may be considered. Our patient underwent aortic coarctation, and ASD and VSD repair at age 14 weeks. At followup echocardiogram at age 7 months, the coarctation had recurred and required balloon dilation. No other complications were present.

\section{Conclusion}

In patients with mediastinal shift on chest radiograph due to suspected lung hypoplasia, MDCT is a non-invasive and appropriate investigation aiding in the diagnosis of congenital pulmonary venolobar syndrome and possible associated cardiac defects.

1. Armstrong P, Wilson AG, Dee P, Hansell DM. Imaging of Diseases of the Chest. 3rd ed. Philadelphia, USA: Mosby, 2004.

2. Kirks DR, Griscom NT. Practical Pediatric Imaging. 3rd ed. Philadelphia, USA: Lippincott Williams and Wilkins, 2006 .

3. Konen E, Raviv-Zilka L, Cohen RA, et al. Congenital pulmonary venolobar syndrome: Spectrum of helical CT findings with emphasis on computerized reformatting. Radiographics 2003;23:1175-1184.

4. Lee EY, Boiselle PM, Cleveland RH. Multidetector CT evaluation of congenital lung anomalies Radiology 2008;247(3):632-648.

5. Cooper G. Case of malformation of the thoracic viscera: Consisting of imperfect development of the right lung and transposition of the heart. London Med Gazette1836;18:600-601.

6. Chassinat R. Observation danomalies anatomiques remarquables de lappareil circulatoire, avec hepatocele congenial, nayant donne lieu pendant la vie a aucun symptom particulier. Arch Gen Med 1836;11:80-84.

7. Halasz NA, Halloran KH, Liebow AA. Bronchial and arterial anomalies with drainage of the right lung into the inferior vena cava. Circulation 1956;14:826-846.

8. Dotter CT, Hardisty NM, Steinberg I. Anomalous right pulmonary vein entering the inferior vena cava: two cases diagnosed during life by angiography and cardiac catheterization. Am J Med Sci 1949;218:3136.

9. Neill CA, Ferencz C, Sabiston DC, Sheldon H. The familial occurrence of hypoplastic right lung with systemic arterial supply and venous drainage "scimitar syndrome". Bull J Hop Hosp 1960;107:1-15.

10. Dillman JR, Yarram SG, Hernandez RJ. Imaging of pulmonary venous developmental anomalies. Am J Roentgenol 2009; 192:1272-1285

11. Lucaya J, Strife JL, Baert AL. Pediatric Chest Imaging. 2nd ed. Berlin: Springer, 2007.

12. Kauczor HU. MRI of the Lung. Berlin: Springer, 2009. 